\title{
Correction of the spin chromaticity in RHIC
}

\author{
J. Kewisch, M. Bai, D. Bruno, \\ F. Meot, T. Roser
}

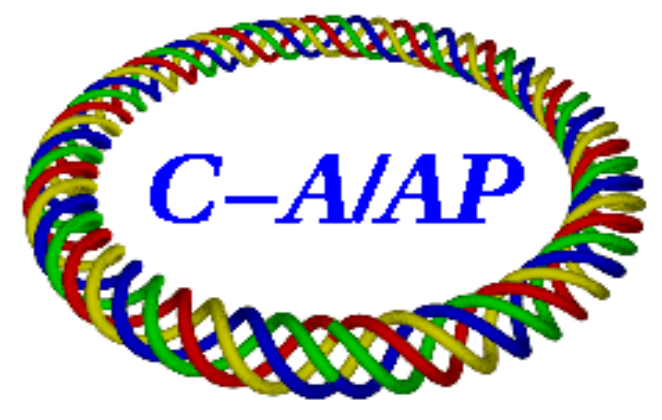

\section{Collider-Accelerator Department Brookhaven National Laboratory Upton, NY 11973}

Notice: This document has been authorized by employees of Brookhaven Science Associates, LLC under Contract No. DE-AC02-98CH10886 with the U.S. Department of Energy. The United States Government retains a nonexclusive, paid-up, irrevocable, world-wide license to publish or reproduce the published form of this document, or allow others to do so, for United States Government purposes. 


\title{
Correction of the Spin Chromaticity in RHIC
}

\author{
J. Kewisch, M. Bai, D. Bruno, F. Meot, T. Roser
}

Abstract

The independence of the spin tune of the momentum is a critical requirement for a full spin flip using the RHIC spin flipper. We show how the spin chromaticity can be corrected with a minimum impact on the RHIC optics and how the correction improves the spin flip.

\section{Introduction}

The RHIC spin flipper is designed to flip the spin helicity of all RHIC bunches in the blue ring in order to eliminate systematic errors in the experiments. Ideally, the proton beam would preserve the polarization during the flip. In reality some loss of polarization is unavoidabler., The spin flip is obtained by the use of five AC dipoles. The tune of the AC dipoles is swept slowly across the spin tune. This rotates the spin rotation axis by 180 degrees and the spin of all particles will follow adiabatically. The efficiency of the spin flip is given by the Froissart-Stora formula:

$$
P_{f}=\left(2 \exp \left(-2 \pi\left|\epsilon_{k}\right|^{2} / \alpha\right)-1\right) P_{i}
$$

where $P_{f}$ and $P_{i}$ are the final and initial polarization, $\epsilon_{k}$ is the resonance strength and $\alpha$ is the sweep rate.

Two Siberian snakes which are installed in RHIC at opposite sides of the ring. The spin tune is

$$
\nu=\frac{1}{2}+\frac{\gamma G\left(\theta_{1}-\theta_{2}\right)}{2 \pi}
$$

where $\theta_{1}$ and $\theta_{2}$ are the integrated bending angles of the first and second arc, respectively. For the on-energy and on-axis proton both $\theta_{1}$ and $\theta_{2}$ are equal $\pi$ (180 Degrees) and the spin tune is $\frac{1}{2}$ independent of the beam energy.

This changes when betatron and synchrotron oscillation are considered [1][2]. Since the transverse oscillations are fast compared to the resonance crossing time their effects average to zero, but we do have to consider the effect of synchrotron oscillations. The change in the bending angles are $\Delta \theta_{1}=\left(x_{1}^{\prime}-x_{2}^{\prime}\right)$ and $\Delta \theta_{2}=\left(x_{2}^{\prime}-x_{1}^{\prime}\right)$ where $x_{1}^{\prime}$ and $x_{2}^{\prime}$ are the slopes of the trajectory at the two snakes. So the spin tune becomes $\frac{1}{2}+2 \gamma G\left(x_{2}^{\prime}-x_{1}^{\prime}\right)$. The $x^{\prime}$ can be expanded in a Taylor series of the momentum of the proton:

$$
x^{\prime}=D 1^{\prime} \frac{\Delta p}{p}+D 2^{\prime}\left(\frac{\Delta p}{p}\right)^{2}+\ldots
$$


where the constant $D 1^{\prime}$ is the slope of the dispersion function and $D 2^{\prime}$ and higher terms describe the non-linear of $x^{\prime}$ and the momentum. With the subscript number indicating the snake we get:

$$
\nu=\frac{1}{2}+\frac{\gamma G}{\pi}\left[\left(D 1_{1}^{\prime}-D 1_{2}^{\prime}\right) * \frac{\Delta p}{p}+\left(D 2_{1}^{\prime}-D 2_{2}^{\prime}\right) *\left(\frac{\Delta p}{p}\right)^{2} \ldots\right]
$$

This change in the spin tune modifies the resonance crossing speed: If the proton momentum increases during the resonance crossing the crossing time is slower and the final polarization is slightly better, if the momentum decreases he crossing time is faster and the final polarization is significantly worse. If the amplitude of the spin tune variation is large enough one protons might even cross the resonance multiple times with significant loss of polarization.

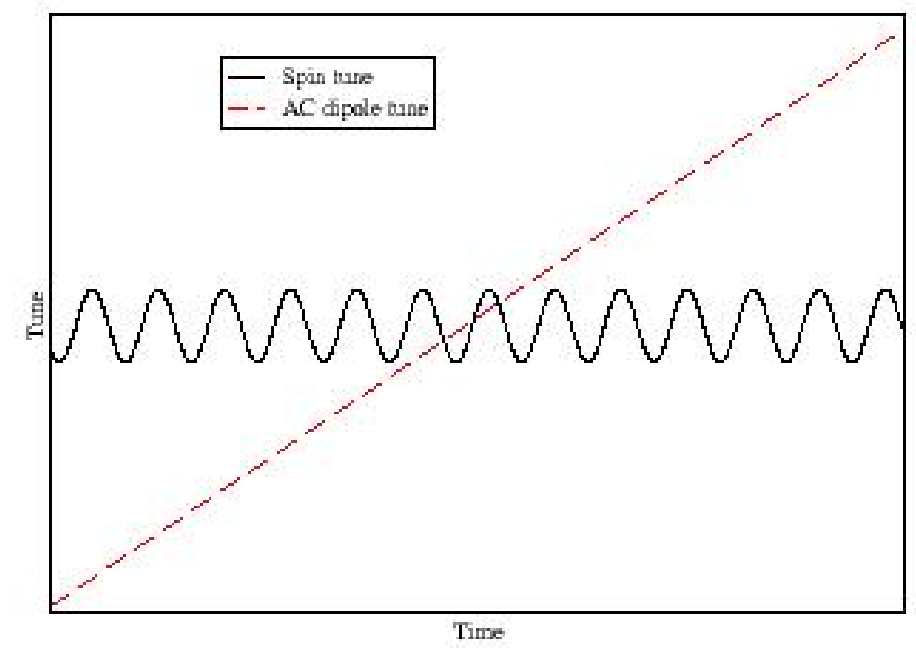

Fig. 1: Mechanism of multiple resonance crossings. The spin tune for an offenergy particle (solid black line) is oscillating with the synchrotron frequency. The AC dipole tune (dashed red line) is ramped across the $\nu=0.5$ line and intersects with the black line three times.

\section{Dispersion prime correction}

RHIC was designed with anti-symmetric interaction regions, which helped in finding solutions for the inner and outer arc optics. With the design optics the difference 
in $D^{\prime}$ is 0.058 . It is impossible to find new settings (within the limitations of the power supplies) that make both $D^{\prime}$ values equal while maintaining all other optical requirements. However, It is possible to modify the optics temporarily for the spin flip and then revert to the design settings for the polarized proton operation.

In this mode the only following requirements remain:

- the betatron tunes must not change to avoid resonant beam loss

- the beta beat should be limited to $25 \%$ to avoid aperture limitations

- the change of quadrupole settings should be minimized to avoid a change of the optics by hysteresis effects.

To change the dispersion one must use quadrupoles that are located in regions where the dispersion is non-zero. In RHIC these are the arc quadrupoles and the Q8 and Q9 quadrupoles. Solutions have been proposed by E. Courant, and D. Trbojevic as well as Chuyu Liu [3]. The limitations of the quadrupole currents make the solution difficult in all these studies.

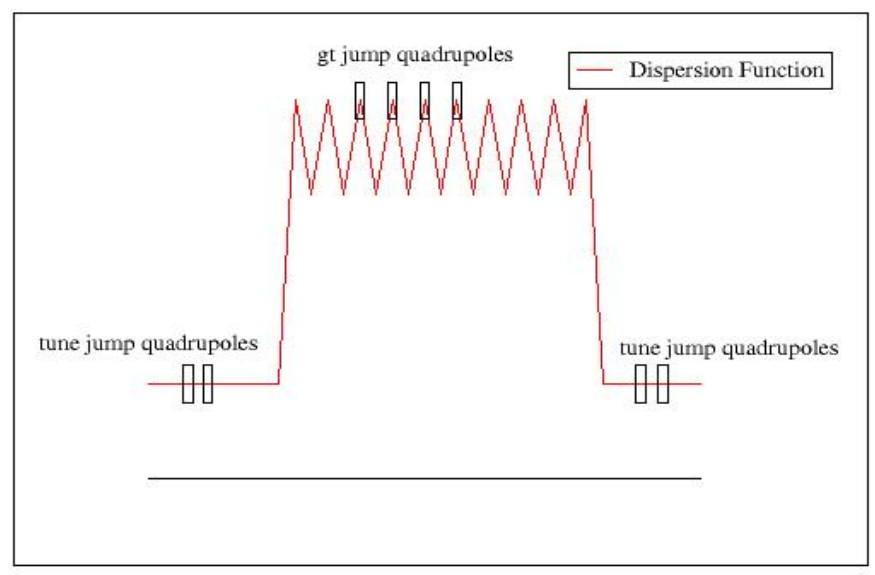

Fig. 2: Layout of the $\gamma_{T}$ quadrupoles in each sextant of RHIC.

In our calculations we used another set of quadrupoles: the $\gamma_{T}$ jump quadrupoles. A set of four quadrupoles are located in each arc and they are used during the acceleration of ions to jump the $\gamma$ of the beam across $\gamma_{T}$. They have identical beta functions, polarity and strength. The horizontal phase advance between them would ideally be 90 degrees, in RHIC it is approximately 82 degrees.

For the change of $\gamma_{T}$ the first and third quadrupole generate a closed bump in the dispersion, and so does the second and forth. In their usual configuration they are not useful to correct the dispersion at the snakes. 


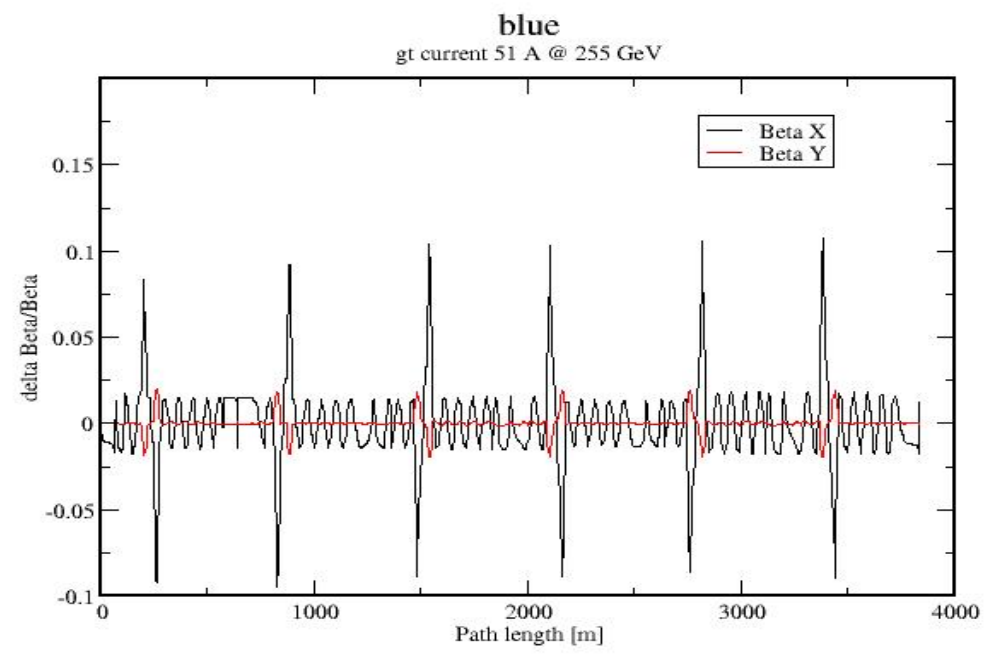

Fig. 3: Relative change of the beta functions. The spikes in $\frac{\Delta \beta}{\beta}$ are in the section between the $\gamma_{T}$ quadrupoles.

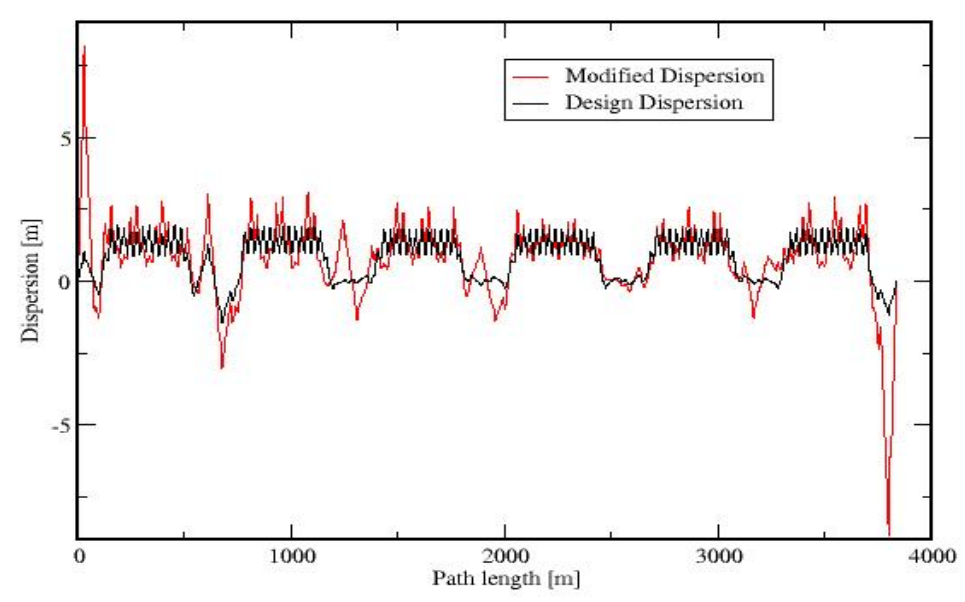

Fig. 4: The dispersion function of the design optics (black) and the dispersion function modified by the $\gamma_{T}$ quadrupoles(red). 
However, the $\gamma_{T}$ quadrupoles are not used during proton operations using the "Elens" optics. If the polarity of two of the quadrupoles is reversed the effect on the dispersion is maximized. The change of the polarity can be accomplished easily by switching the wires at the connector box above the quadrupoles (Figure 5).

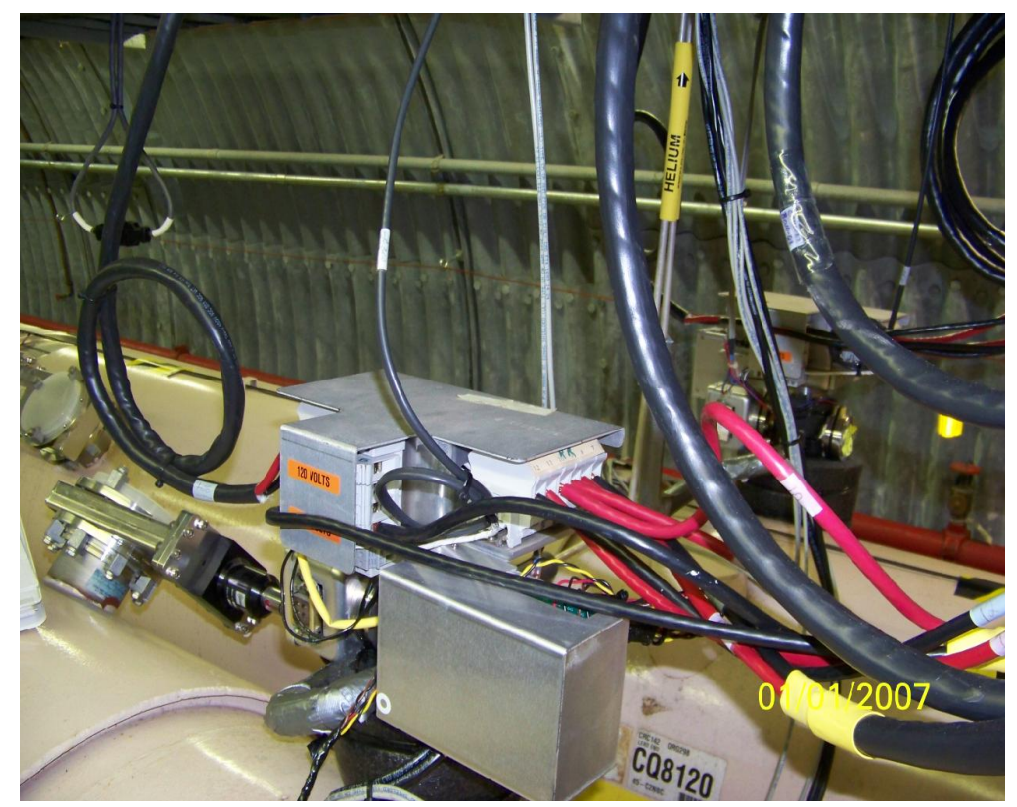

Fig. 5: The connector box on top of the $\gamma_{T}$ quadrupoles allows easy reversal of the polarity.

Since the beta function is the same for all gamma quadrupoles the tune does not change with the quadrupole strength and there is a significantly compensation of the beat beat outside the region of gamma $_{T}$ quadrupoles. Another pair of jump quadrupoles is located at each end of the arc, where the dispersion is zero. These are used during the $\gamma_{T}$ jump to keep the turns constant, but they are not used for our purpose. We power all $\gamma_{T}$ quadrupoles with the same current and chose the polarities so that the difference in $D^{\prime}$ at the snake is minimized. For the E-lens optics we need 55 Amperes at $255 \mathrm{GeV}$ in the Blue ring and 62 Amperes in the yellow ring. The current power supplies are rated for 60 Amperes, but all but one gt quadrupole can be powered up to 100 Amperes. Bigger power supplies without the jump capacity could be added in the future in the yellow ring. With such power supplies it may also be possible to avoid the spikes in the dispersion function close to the STAR interaction region (Figure 4 ).

Figure 6 shows the spin tune vs. momentum deviation before and after correction. The correction reduces the spin tune spread by two orders of magnitude. There is however a non-linear contribution and the tracking results shown below show that this reduces the efficiency of the spin flip for large momentum deviations.

A second order correction might be possible using sextupoles, but the following needs tow be considered:

- The sextupoles are currently powered in four families to correct the first and 

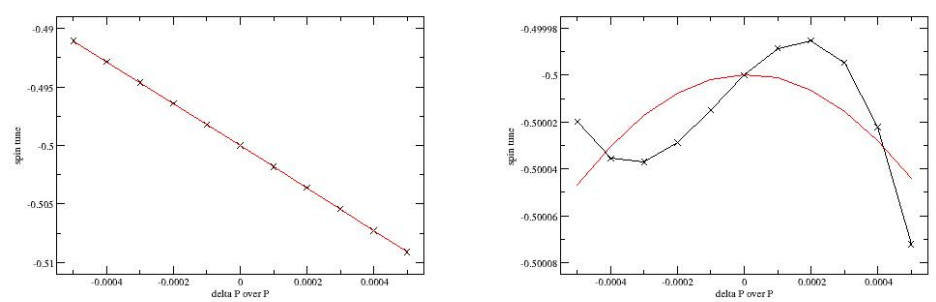

Fig. 6: Spin tune before and after correction. The black curve shows the tunes calculated with ZGOUBI, the red curve is a parabola fitted to the values.

second order chromaticity. Any correction scheme would still have to perform this task.

- Like the $\gamma_{T}$ quadrupoles the sextupoles are currently powered to minimize the effect on the betatron oscillations and the dispersion. For a spin tune correction the polarity of some sextupoles must be changed. This may negatively affect the dynamic aperture of the machine.

As we see below a satisfactory spin flip might be achieved without such correction.

\section{The layout of the RHIC spin flipper}

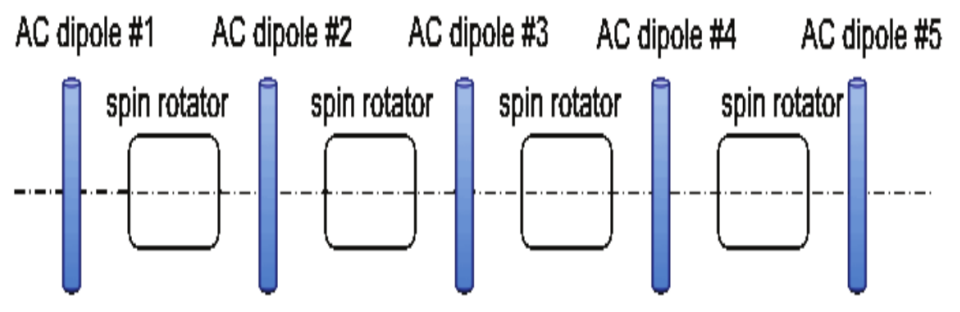

Fig. 7: Layout of the Spin flipper.

The spin flipper consist of four DC dipoles with horizontal deflection and five AC dipoles with vertical deflection as shown in Figure 7. The DC dipoles rotate the spin by 45 degrees. The AC dipoles build two closed orbit bumps where the middle AC dipole is used for both bumps. The second bump is shifted in time by 45 degrees. In our simulations we have split the middle AC dipole in half, so that the first half is powered with the first bump, the second half with the second bump. The strength of the first and last AC dipole is 0.038 kGauss, the strength of the others are calculated to close the bumps.

The Resonance strength for this arrangement is:

$$
\epsilon_{k}=\Phi_{A C} \sin \left(\Phi_{D C}\right) \sin \left(\Phi_{D C} / 2\right) / \pi
$$


where $\Phi_{A C}$ is the peak spin rotation of the 2 nd $A C$ dipole and $\Phi_{D C}$ is the spin rotation of the DC dipole. The sweep rate is:

$$
\alpha=\frac{\Delta \nu}{2 \pi N}
$$

where $\Delta \nu=0.02$ is the frequency range of the sweep and $N$ is the number of turns for the sweep.

\section{Spin Tracking}

The spin flip has been simulated using the tracking program ZGOUBI [4] in various configuration. The results presented here are obtained for a beam energy of $255 \mathrm{Gev}$, corresponding to $\gamma G=486.75$. The RHIC beam has a longitudinal emittance of 1.5 $\mathrm{eV} \cdot \mathrm{s}$ (This describes the edge of the beam).

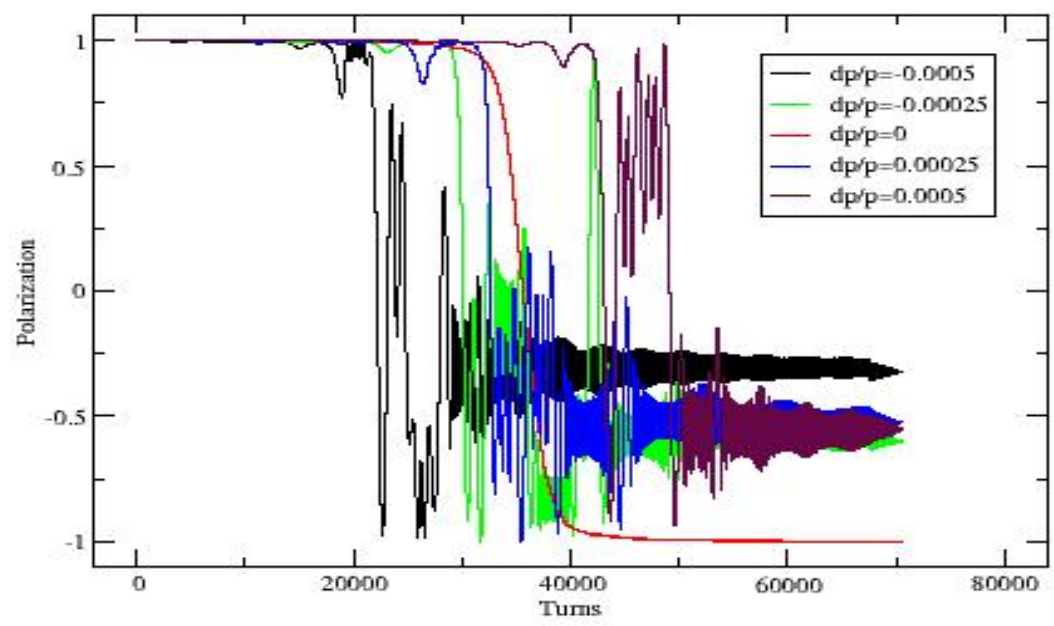

Fig. 8: Spin flip using the $28 \mathrm{MHz}$ cavity system without the $D^{\prime}$ correction for varying synchrotron oscillation amplitude.

The first two calculations use the $28 \mathrm{MHz}$ RF system for bunching of the beams. The total acceleration voltage is set to $50 \mathrm{kV}$ and the energy spread is $2.7 \cdot 10^{-4}$. Five particles starting on axis with the momentum of $0.0, \pm 2.5 \cdot 10^{-4}$ and $\pm 5 \cdot 10^{-4}$ where tracked through the resonance.

Figure 8 shows the vertical spin component without the $D^{\prime}$ correction. While the on-energy particle flips the spin as predicted by Froissart-Stora, the off-energy particles are depolarized. Figure 9 shows the same setup with the $\gamma_{T}$ quadrupoles turned on. Here the particles with an energy spread of $\pm 2.5 \cdot 10^{-4}$ have a satisfactory flip, the 


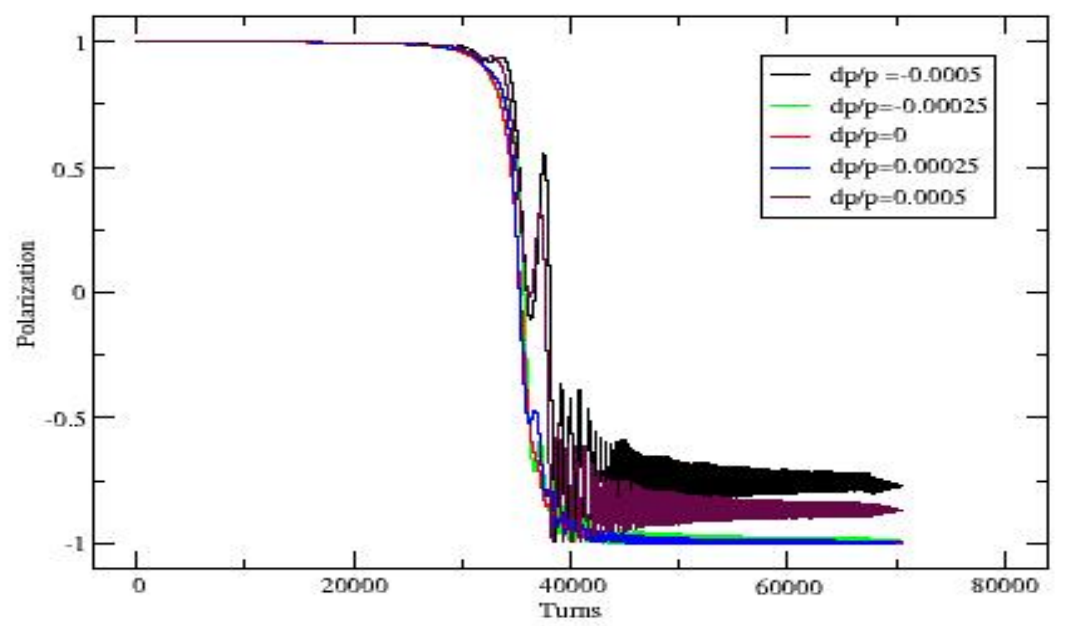

Fig. 9: Spin flip using the $28 \mathrm{MHz}$ cavity system with the $D^{\prime}$ correction.

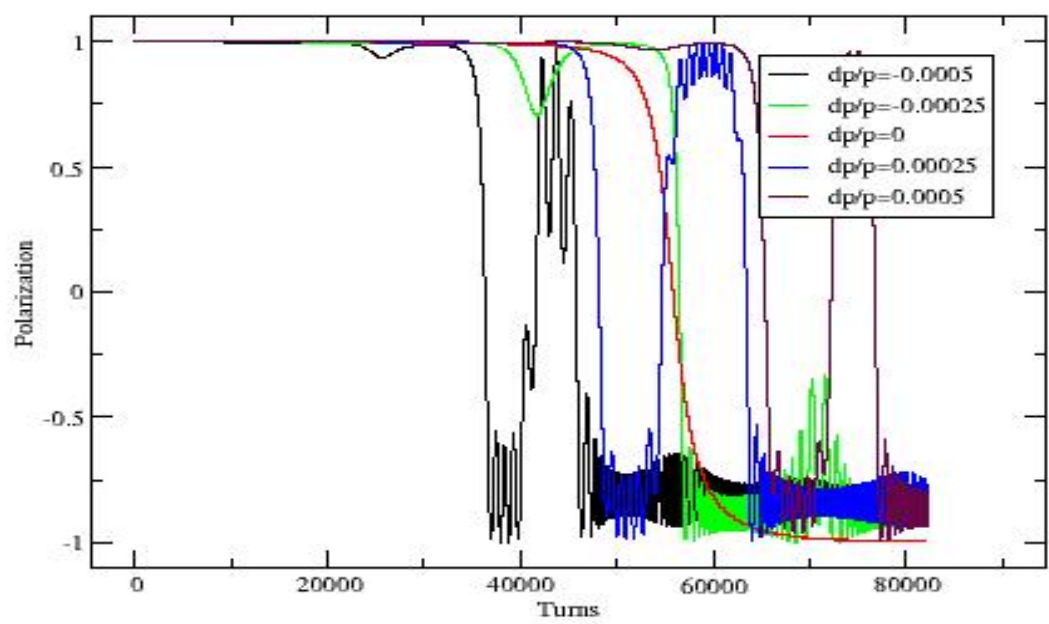

Fig. 10: Spin flip using the $9 \mathrm{MHz}$ cavity system without the $D^{\prime}$ correction. 
particles with $\pm 5 \cdot 10^{-4}$ energy spread are still depolarized. It is questionable if the $28 \mathrm{MHz}$ system can be operated stably at this low voltage. With a voltage of $100 \mathrm{kV}$ the energy spread would be $3.2 \cdot 10^{-4}$.

By using the $9 \mathrm{MHz}$ RF system with a total voltage of $20 \mathrm{kV}$ the energy spread of the beams is lowered to $1.6 \cdot 10^{-4}$ In the calculation without the correction the multiple crossings of the resonance are clearly visible (Figure 10). With the $\gamma_{T}$ quadrupoles turned on all particles perform a satisfactory spin flip (Figure 11).

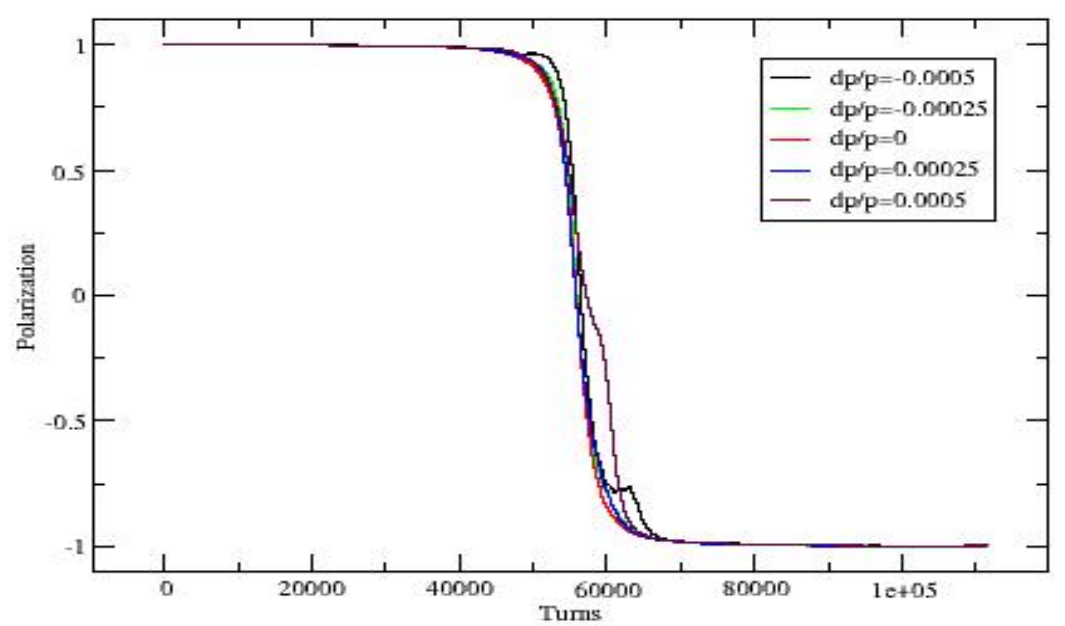

Fig. 11: Spin flip using the $9 \mathrm{MHz}$ cavity system with the $D^{\prime}$ correction.

Finally we include also the transverse oscillations of the beam (Figure 12). Here we track 80 particle were tracked. For each of the above used energy deviations the $x$ and $y$ coordinated were set to $0.1 \mathrm{~mm}$ and the $x^{\prime}$ and $y^{\prime}$ coordinates were set to 0.2 mrad (The beta function at the start point is $0.6 \mathrm{~m}$ in both directions.) The overlay of the vertical spin component of all 80 particles is shown in black, the average is shown in red. The final polarization is $98 \%$. 


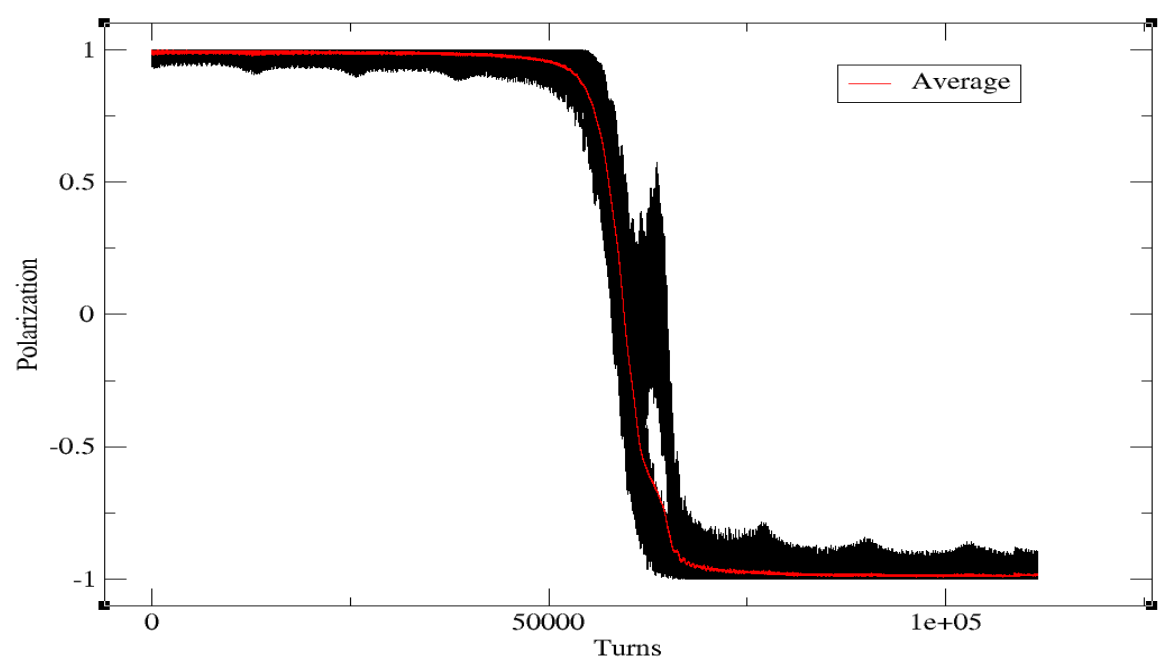

Fig. 12: Spin flip including betatron oscillations. The overlay of the vertical spin component of all 80 particles is shown in black, the average is shown in red.

\section{References}

[1] M. Bai et al.: Impact on Spin Tune From Horizontal Orbital Angle Between Snakes and Orbital Angle Between Spin Rotators, C-A/AP/\#334

[2] M. Bai et al.: RHIC Spin Flipper Status and Simulation Studies, PAC 2011, New York, MOP191

[3] C. Liu: to be published at IPAC 2013

[4] http://sourceforge.net/projects/zgoubi/ 